\title{
Choriocarcinoma: a rare case of stomach metastasis
}

\author{
Raju Agarwal $^{1}$, Col Dharmesh ${ }^{2}$, Madhusudan Dey $^{1}$, Charu D. Pawar ${ }^{1}$
}

${ }^{1}$ Department of Obstetrics \& Gynaecology, AFMC, Pune, India
${ }^{2}$ Department of Medical Oncology, CH(SC), Pune, India

Received: 13 June 2014

Accepted: 1 July 2014

\section{*Correspondence:}

Dr. Madhusudan Dey,

E-mail: deym1@yahoo.com

(C) 2014 Agarwal R et al. This is an open-access article distributed under the terms of the Creative Commons Attribution Non-Commercial License, which permits unrestricted non-commercial use, distribution, and reproduction in any medium, provided the original work is properly cited.

\begin{abstract}
Choriocarcinoma is a rare form of cancer which commonly occurs in women of reproductive age, rarely in postmenopausal women and in women under 20 years of age. We report a rare case of uterine choriocarcinoma with stomach metastasis in a 29 year-old woman who presented with upper gastrointestinal symptoms. The presented case report emphasizes the need for innovative treatment approach and appropriate diagnostic technology to enable early diagnosis and correct treatment. Furthermore the case highlights the need for healthcare workers to consider rare causes of gastrointestinal signs and symptoms.
\end{abstract}

Keywords: Choriocarcinoma, stomach metastasis, Hydatidiform mole, EMA-CO, Bhcg

\section{INTRODUCTION}

Chriocarcinoma also known as trophoblastic tumour is a rare form of malignancy which occurs in the female genital tract and is commonly associated with pregnancy. It may develop after a normal pregnancy; however, it is usually associated with molar pregnancy, ectopic pregnancy, miscarriage, or abortion. ${ }^{1}$ It has an incidence of about 1 in 30000 pregnancies. It should be considered when persistent bleeding follows any pregnancy event. ${ }^{2}$ In most cases chriocarcinoma is associated with a positive pregnancy test even without pregnancy and with high levels of beta human chorionic gonadotrophic hormone (Bhcg).

\section{CASE REPORT}

29 years old para 3 women reported with symptoms of breathlessness, easy fatigability of three months duration and passing of dark tarry stools of 15 days duration. Her past history revealed a suction \& evacuation done for molar pregnancy 3 years back with no follow up thereafter. The general physical examination revealed extreme pallor, pulse 110/min, BP $90 / 60 \mathrm{~mm}$ of $\mathrm{Hg}$. Systemic examinations was normal. Gynaecological examination did not show any lesion on vulva, vagina or cervix. The uterus was anteverted and normal size.

The investigations done revealed a $\mathrm{Hb} 2.5 \mathrm{gm} \%$, PCV $11 \%$, platelet count $200000 / \mathrm{cmm}$, LFT \& RFT were normal, stool for occult blood was positive and Bhcg was 177760 IU/L. Chest $X$ ray showed a suspicious subpleural nodule on left side. A complete metastatic workup was done. CECT chest revealed a subpleural nodule (Figure 1). CT abdomen and pelvis revealed multiple heterogeneously enhancing hypodense lesions in segment 5,6,7,8 of Rt lobe liver $(2-3.4 \mathrm{~cm})$, Uterus \& Ovaries were normal (Figure 2). CT Brain was normal. Upper gastro intestinal endoscopy revealed a $1 \times 1 \mathrm{~cm}$ protuberant lesion with central blood vessel in the body of the stomach (Figure 3).

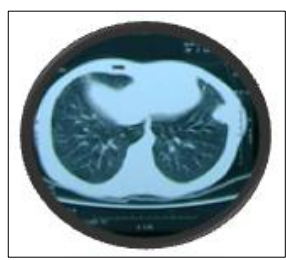

Figure 1: CECT chest showing subpleural nodule. 


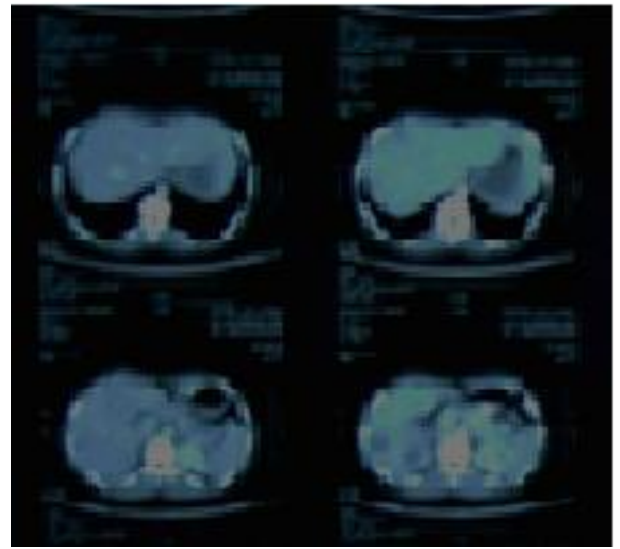

Figure 2: Multiple hypodense lesion in liver.

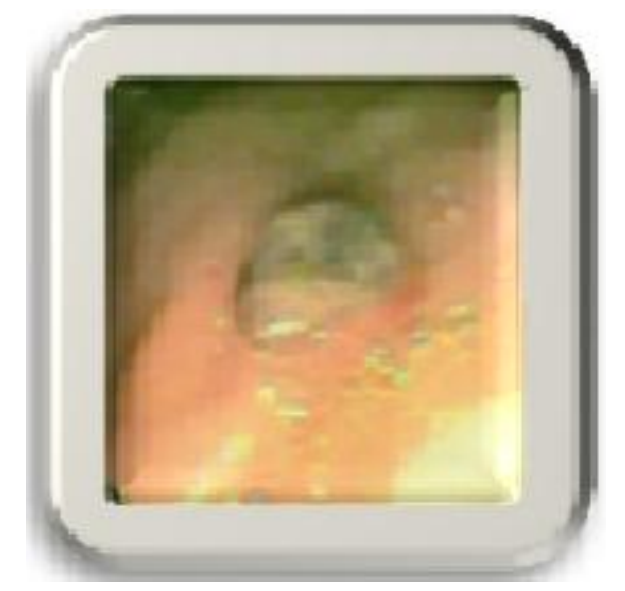

Figure 3: Endoscopy showing 1x1 cm lesion in stomach.

In view of the medical history, clinical examination, laboratory and radiological investigations a final diagnosis of metastatic choriocarcinoma stage IV C (high risk with WHO prognostic score of >8) was arrived at.

Despite repeated blood transfusions her haemoglobin could not be raised to desired levels for the standard EMA-CO regimen (E-etoposide, M-methotrexate, Aactinomycin, C-cyclophospamide, O-vincristine). This was due to the actively bleeding gastrointestinal lesion. To overcome this problem a innovative approach was attempted. She was started on single agent methotrexate $(1.1 \mathrm{mg} / \mathrm{kg})$ with the hope that the lesion would stop bleeding. The bleeding stopped within 48 hours as evidence by rise in haemoglobin levels with subsequent blood transfusions. Thereafter she was started on EMA$\mathrm{CO}$ regimen (omitting methotrexate in first cycle). The BHCG fell to $188 \mathrm{IU} / \mathrm{L}$ before second cycle. Bhcg was undetected before third cycle of EMA-CO. She received total three cycles of chemotherapy as consolidation after Bhcg was undetectable making a total of five cycles. She was given a total of 20 packed red blood cells $\& 8$ fresh frozen plasma over a period of 30 days. The post chemotherapy follow up has been for 5 years now and the patient is disease free.

\section{DISCUSSION}

Choriocarcinoma is an extremely malignant tumour, may be considered a carcinoma of the chorionic epithelium. Choriocarcinoma may arise from any type of pregnancy, including a normal term pregnancy and from homozygous or heterozygous complete mole. ${ }^{3}$ Choriocarcinoma is suspected when there is abnormal uterine bleeding following an abortion or hydatidiform mole. This is one of the most frequent presentations of choriocarcinoma.

Not all patients have a demonstrable lesion in the uterus after an intrauterine gestation and at times, symptoms related to the metastasis are the first presentation. Metastasis of choriocarcinoma occurs most frequently in the lungs, brain and liver. The gastrointestinal tract can also be affected in less than $5 \%$ cases. There are few reports of metastatic choriocarcinoma affecting the gastrointestinal tract. It can present in the form of upper gastrointestinal bleeding, intussusception, ileal perforation or rectal bleeding. ${ }^{4}$

Our case presented at a rare site in stomach with no evidence of disease in the uterus. A high index of suspicion in all childbearing age women can detect this rare malignancy with bizarre symptom presentation not related to genital tract as was in our case and a simple test of Bhcg in the workup of women with radiological evidence of systemic metastasis with unknown primary will help in saving these young women.

High risk choriocarcinoma, WHO prognostic score $>7$ is treated with EMA-CO regime with a cure rate between 67 and 85 percentage., 5 These women should be managed in centers with expertise to deal with such cases as future fertility may also be an issue in young women. Our case despite being in poor general condition on presentation could be saved due to timely diagnosis and appropriate chemotherapy thus achieving complete remission.

The specific treatment of bleeding gastrointestinal lesion is controversial. The modalities of treatment suggested are surgery and angiographic embolization ${ }^{7}$ without any survival benefit with these approaches. Our case presented with upper gastro intestinal bleed and the facilities of angiographic embolization did not exist at that time in the institution. So we tried an innovative approach to start with methotrexate as it less bone marrow suppressive. We could achieve the desired result of stopping upper GI bleed and thereafter give combination chemotherapy. We did not find this type of management approach on literature search. We recommend that in desperate situations an innovative individualized treatment may be life saving.

Funding: No funding sources

Conflict of interest: None declared

Ethical approval: Not required 


\section{REFERENCES}

1. Nugent D, Hassadia A, Everrard J et al. Postpartum chriocarcinoma. Presentation, management and survival. J Reprod Med. 2006;51:819.

2. Soper JT. Gestational trophoblastic disease. Obstet Gynaecol. 2006;108:176.

3. Fisher RA, Lower SD, Povey S, Bagshawe KD. Genetically homozygous choriocarcinoma following pregnancy with hytaditiform mole. $\mathrm{Br} \mathrm{J}$ Cancer. 1988;58:788-892.

4. Chaturvedi M, Vaideeswar P, Pandit A. Metastatic choriocarcinoma: an unusual cause of severe anaemia. J Postgrad Med. 2005;51:230-1.
5. Lurain JR, Singh DK, Schink J. Primary treatment of metastatic high risk gestational trophoblastic neoplasia with EMA-CO chemotherapy. J Reprod Med. 2006a;51:767.

6. Gulia S, Bajpai J, Gupta S, Maheshwari A et al. Outcome of gestational trophoblastic neoplasia: experience from tertiary care center in India. Clin Oncol (R Coll Radiol). 2014 Jan;26(1):39-44.

7. Rosenblatt GS, Walsh CJ, Chung S. Metastatic testicular tumour presenting as gastrointestinal haemorrhage. J Urol. 2000;164:1655.

DOI: 10.5455/2320-1770.ijrcog20140924

Cite this article as: Agarwal R, Col D, Dey M,

Pawar CD. Choriocarcinoma: a rare case of stomach metastasis. Int J Reprod Contracept Obstet Gynecol 2014;3:787-9. 